\title{
Investigating the Impact of a Genome-Wide Supported Bipolar Risk Variant of MADILI on the Human Reward System
}

\author{
Sarah Trost ${ }^{*, 1}$, Esther K Diekhof ${ }^{1,2}$, Holger Mohr ${ }^{1,3}$, Henning Vieker ${ }^{1,4}$, Bernd Krämer ${ }^{1,5}$, Claudia Wolf ${ }^{1,6}$, \\ Maria Keil', Peter Dechent ${ }^{7}$, Elisabeth B Binder ${ }^{8}$ and Oliver Gruber ${ }^{1,5}$ \\ 'Department of Psychiatry and Psychotherapy, Center for Translational Research in Systems Neuroscience and Clinical Psychiatry, Georg August \\ University Goettingen, Goettingen, Germany; ${ }^{2}$ Biocenter Grindel and Zoological Institute, Department of Human Biology, Hamburg University, \\ Hamburg, Germany; ${ }^{3}$ Department of Psychology, Neuroimaging Center and Institute of General Psychology, Biopsychology, and Methods of \\ Psychology, Technische Universitaet Dresden, Dresden, Germany; ${ }^{4}$ Department of Psychiatry and Psychotherapy, University Medical Center, \\ Eppendorf, Hamburg, Germany; ${ }^{5}$ Section for Experimental Psychopathology and Neuroimaging, Department of General Psychiatry, Heidelberg \\ University Hospital, Ruprecht Karls University, Heidelberg, Germany; 'aboratory of Behavioral Neuroscience, Biomedical Research Center, \\ National Institute on Aging, Baltimore, MD, USA; ${ }^{7}$ Department of Cognitive Neurology, Georg August University, Goettingen, Germany; \\ ${ }^{8}$ Department of Translational Psychiatry, Max Planck Institute of Psychiatry, Munich, Germany
}

Recent genome-wide association studies have identified MADILI (mitotic arrest deficient-like I) as a susceptibility gene for bipolar disorder and schizophrenia. The minor allele of the single-nucleotide polymorphism (SNP) rs I I 764590 in MADILI was associated with bipolar disorder. Both diseases, bipolar disorder and schizophrenia, are linked to functional alterations in the reward system. We aimed at investigating possible effects of the MADILI rs I 1764590 risk allele on reward systems functioning in healthy adults. A large homogenous sample of 224 young (aged I8-3I years) participants was genotyped and underwent functional magnetic resonance imaging ( $\mathrm{MMRI}$ ). All participants performed the 'Desire-Reason Dilemma' paradigm investigating the neural correlates that underlie reward processing and active reward dismissal in favor of a long-term goal. We found significant hypoactivations of the ventral tegmental area (VTA), the bilateral striatum and bilateral frontal and parietal cortices in response to conditioned reward stimuli in the risk allele carriers compared with major allele carriers. In the dilemma situation, functional connectivity between prefrontal brain regions and the ventral striatum was significantly diminished in the risk allele carriers. Healthy risk allele carriers showed a significant deficit of their bottom-up response to conditioned reward stimuli in the bilateral VTA and striatum. Furthermore, functional connectivity between the ventral striatum and prefrontal areas exerting top-down control on the mesolimbic reward system was reduced in this group. Similar alterations in reward processing and disturbances of prefrontal control mechanisms on mesolimbic brain circuits have also been reported in bipolar disorder and schizophrenia. Together, these findings suggest the existence of an intermediate phenotype associated with MADILI.

Neuropsychopharmacology (2016) 4I, 2679-2687; doi: I0.1038/npp.2016.70; published online 8 June 2016

\section{INTRODUCTION}

Bipolar disorder and schizophrenia are severe chronic and highly heritable psychiatric disorders. Family, twin and adoption studies have provided strong evidence for a substantial familial overlap with both bipolar disorder and schizophrenia, suggesting partially shared genetic underpinnings of these diseases (Cardno and Owen, 2014).

Through the use of large genome-wide association studies (GWAS), the search for genetic risk loci for bipolar disorder and schizophrenia has further moved forward (Zhang et al, 2012). Recently, Cichon et al (2011) identified MAD1L1

\footnotetext{
*Correspondence: Dr S Trost, Department of Psychiatry and Psychotherapy, Center for Translational Research in Systems Neuroscience and Clinical Psychiatry, Georg August University Goettingen, Von-SieboldStrasse 5, Goettingen 37075, Germany, Tel: +49 55I 39 66179/666I5, Fax: +4955 39 9337, E-mail: s.trost@med.uni-goettingen.de

Received 12 December 2015; revised 3 May 2016; accepted 5 May 2016; accepted article preview online 13 May 2016
}

(mitotic arrest deficient-like 1) as a potential susceptibility factor for bipolar disorder in a GWAS. The minor allele of the single-nucleotide polymorphism (SNP) rs11764590 was significantly associated with bipolar disorder. Subsequent GWAS also found significant associations of MAD1L1 with bipolar disorder (Schizophrenia Psychiatric Genome-Wide Association Study (GWAS) Consortium, 2011) as well as associations with both bipolar disorder and schizophrenia (Bergen et al, 2012; Ruderfer et al, 2014).

MAD1L1 [OMIM 602686] with the intronic SNP rs11764590 is located on chromosome 7p22.3 and is expressed in numerous human tissues (Sun et al, 2013; Tsukasaki et al, 2001). In the brain, MAD1L1 expression is measurable in cortical and subcortical areas including the basal ganglia (including the dorsal and ventral striatum), the ventral tegmental area (VTA) (Hawrylycz et al, 2012), and the hippocampus (Cichon et al, 2011; 'GTEx Portal', n.d.; http://www.gtexportal.org/home/gene/ MAD1L1), ('Microarray Data :: Allen Brain Atlas: Human Brain', n.d.; http://human.brain-map.org/microarray/search/show?exact_ 
match $=$ false\&search_term $=$ MAD1L1\&search_type = gene) .

MAD1L1 is a checkpoint gene; it is involved in regulating the spindle assembly checkpoint during mitosis. Variable gene expression levels and mutations of the MAD1L1 gene are associated with chromosomal instability and have a role in carcinogenesis and aging (Cichon et al, 2011; Sun et al, 2013; Tsukasaki et al, 2001). Recently, it was shown that experimental manipulation of the transcription of MIR137, another leading candidate schizophrenia susceptibility gene (Schizophrenia Psychiatric Genome-Wide Association Study (GWAS) Consortium, 2011), was followed by altered expression levels of MAD1L1 in a neural cell line (Hill et al, 2014). These findings suggest common molecular pathways through which genetic variation in these susceptibility genes could confer the risk for schizophrenia or bipolar disorder (Hill et al, 2014). Another protein that has been shown to influence MAD1L1 gene expression is the Cockayne syndrome complementation B (CSB) protein (Lake et al, 2014). Mutations in CSB are accountable for the majority of Cockayne syndrome cases, an inherited premature aging disease linked to numerous developmental and neurological deficits such as microcephaly, hypomyelination, calcification and neuronal loss, mental retardation, ataxia, and intellectual decline (Lake et al, 2014; Vessoni et al, 2016). This potentially regulatory effect of CSB on MAD1L1 expression possibly indicates a role of MAD1L1 in the central nervous system.

Bipolar disorder and schizophrenia do not only partially share a genetic basis, but also show phenotypic similarities, eg, with regard to symptomatology (Psychiatric GWAS Consortium Bipolar Disorder Working Group, 2011; Tamminga et al, 2013). Moreover, both disorders are characterized by altered functional brain activation patterns in prefrontal and subcortical networks (Calhoun et al, 2011; Chai et al, 2011), including the reward system (Deserno et al, 2013; Gradin et al, 2011; Whalley et al, 2012).

The reward system in the brain is based on a neural circuitry including regions of the mesolimbic dopamine system, in particular the ventral striatum and VTA (Diekhof and Gruber, 2010). In a recent functional magnetic resonance imaging (fMRI) study, we found that euthymic to mildly depressed bipolar I patients showed a reduced bottom-up responsiveness of the ventral striatum and a disturbed top-down control of the mesolimbic reward system by prefrontal brain regions while performing a specific reward paradigm, the 'Desire-Reason Dilemma' paradigm (DRD paradigm) (Trost et al, 2014). The DRD paradigm assesses the neural mechanisms underlying reward processing and active reward dismissal in favor of a long-term goal (Diekhof and Gruber, 2010). With regard to schizophrenia, recent fMRI studies showed that schizophrenic patients also exhibited abnormal subcortical reward processing (Juckel et al, 2006; Subramaniam et al, 2015) and associated alterations in functional connectivity within the salience network and reward regions (Gradin et al, 2013). Similar effects with regard to the ventral striatum were shown in healthy first-degree relatives of schizophrenic patients (Grimm et al, 2014). As already similarly reported for prodromal, drug-naive and chronic schizophrenia patients (Deserno et al, 2013), these first-degree relatives exhibited an attenuated striatal response during reward anticipation (Grimm et al, 2014).
Imaging genetics is a promising approach to shed light on the neurophysiological impact of susceptibility genes on the human brain. It offers the possibility to investigate the genetic effects on the neuronal level that may mediate the vulnerability to psychiatric disorders in the sense of an intermediate phenotype (Gottesman and Gould, 2003).

In the present study, we aimed to investigate the effects of the MAD1L1 risk allele on the neurofunctional level. To this end, a homogenous group of healthy adults underwent fMRI. All participants performed the DRD paradigm to investigate possible gene effects of the MAD1L1 risk allele on the mesolimbic reward system and other brain regions. We hypothesized that risk allele carriers would show alterations in subcortical reward processing similar to those found in bipolar and schizophrenic patients as well as in their first-degree relatives according to the concept of an intermediate phenotype.

\section{MATERIALS AND METHODS}

\section{Subjects}

Participants in the Genomic Imaging Goettingen (GIG) study $(n=299)$ were recruited by advertisements in intern online student networks and local newsletters in the GeorgAugust-University Goettingen and the University Medical Center Goettingen. Healthy young adults aged 18-31 years were included. Exclusion criteria were past or present psychiatric disorders according to ICD-10, a positive family history of psychiatric disorders, substance abuse during the last month, cannabis abuse during the last 2 weeks, mental retardation, dementia, neurological or metabolic diseases, and pregnancy in women. All participants were Caucasians with European ancestry.

Imaging and performance data for at least $70 \%$ of all trials, and MAD1L1 rs11764590 genotype were available for 224 participants (exclusion criteria were unavailability of genotype in 12 cases, failure of fMRI data acquisition in 7 cases, and fMRI motion artifacts and/or less than $70 \%$ correct answers across all task conditions in 56 cases).

The mean age of the participants was 24.01 years $( \pm 2.45$ years; range 18-31 years), 212 subjects were right handed, 12 were left handed or both ( 1 case ambidextrous), and 87 subjects were male. All participants had passed a higher general school qualification.

The Temperament and Character Inventory (TCI) (Cloninger et al, 1998) and the Barratt Impulsiveness Scale 11 (BIS) (Patton et al, 1995) were performed by all participants to assess personality traits associated with dopaminergic neurotransmission and reward-related brain activation. Both scales investigate aspects of personality traits characteristic for bipolar disorder, eg, enhanced impulsivity (Strakowski et al, 2010). TCI and BIS data were available for 220 participants.

All participants provided written informed consent after the study procedure had been fully explained. The study was carried out in accordance with the latest version of the Declaration of Helsinki and was approved by the local ethics committee.

\section{Experimental Procedure/DRD Paradigm}

Initially, participants underwent an operant conditioning task. Eight differently colored squares were presented as 
Table I Demographic Variables and Personality Traits

\begin{tabular}{|c|c|c|c|c|}
\hline & $\begin{array}{c}\text { All } \\
\text { participants }\end{array}$ & $\begin{array}{c}\mathrm{C} / \mathrm{C} \\
\text { homozygotes }\end{array}$ & $\begin{array}{c}\mathrm{C} / \mathrm{T} \text { and } \mathrm{T} / \mathrm{T} \\
\text { carriers }\end{array}$ & $P$-values \\
\hline Number & 224 & 131 & 93 & \\
\hline Age & $24.01 \pm 2.45$ & $24.20 \pm 2.51$ & $23.74 \pm 2.35$ & 0.165 \\
\hline Gender & $87 \mathrm{M} / \mathrm{I} 37 \mathrm{~F}$ & $57 \mathrm{M} / 74 \mathrm{~F}$ & $30 \mathrm{M} / 63 \mathrm{~F}$ & 0.086 \\
\hline Handedness & $212 \mathrm{R} / 12 \mathrm{~L}$ & I24 R/7 L & $88 \mathrm{R} / 5 \mathrm{~L}$ & 0.991 \\
\hline Nicotine $^{a}$ & 17 yes/207 no & II yes/I20 no & 6 yes $/ 87$ no & 0.582 \\
\hline Caffeine $^{a}$ & 54 yes/I70 no & 34 yes/97 no & 20 yes/73 no & 0.440 \\
\hline Alcohol $^{a}$ & 0 yes/224 no & 0 yes/131 no & 0 yes/93 no & \\
\hline BIS total & $62.20 \pm 8.44$ & $62.43 \pm 7.98$ & $61.88 \pm 9.08$ & 0.645 \\
\hline $\begin{array}{l}\text { BIS non- } \\
\text { planning }\end{array}$ & $23.64 \pm 4.29$ & $23.64 \pm 3.94$ & $23.65 \pm 4.75$ & 0.990 \\
\hline BIS congitive & $22.31 \pm 3.59$ & $22.57 \pm 3.60$ & $21.96 \pm 3.58$ & 0.208 \\
\hline BIS motor & $16.24 \pm 2.97$ & $16.21 \pm 2.75$ & $16.28 \pm 3.26$ & 0.873 \\
\hline $\mathrm{TCl}$ persistance & $4.70 \pm 2.14$ & $4.79 \pm 2.12$ & $4.57 \pm 2.17$ & 0.446 \\
\hline $\begin{array}{l}\mathrm{TCl} \text { reward } \\
\text { dependence }\end{array}$ & $16.55 \pm 3.42$ & $16.70 \pm 3.37$ & $|6.34 \pm 3.5|$ & 0.439 \\
\hline $\begin{array}{l}\mathrm{TCl} \text { harm } \\
\text { avoidance }\end{array}$ & $13.64 \pm 6.16$ & $12.98 \pm 5.97$ & $14.55 \pm 6.33$ & 0.065 \\
\hline $\mathrm{TCl}$ novelty & $21.32 \pm 5.76$ & $21.62 \pm 5.68$ & $20.90 \pm 5.89$ & 0.368 \\
\hline
\end{tabular}

Abbreviations: BIS, Barratt Impulsiveness Scale; F, female; L, left handed; M, male; $\mathrm{R}$, right handed; $\mathrm{TCl}$, Temperament and Character Inventory.

Data are presented as mean \pm standard deviation.

aNicotine, caffeine, or alcohol use during the last $2 \mathrm{~h}$ before fMRI.

stimuli on a monitor in a shuffled mode. Subjects were instructed to respond to each of the stimuli by button press with their right hand. Button choice was free and subjects were encouraged to explore the stimulus-response-reward contingencies. By doing so, subjects were conditioned to associate two colors (red and green) with an immediate reward (bonus of +10 points), while the other six colors were associated with a neutral outcome. The goal of this operant conditioning task was to establish stimulus-response-reward contingencies for the next phase of the experiment.

Subsequently, subjects were familiarized with the actual experimental task, the DRD paradigm, a delayed matching to sample task. Subjects had to perform blocks of four or eight trials. At the beginning of every block, subjects were shown two targets (two different neutral colors, not the previously conditioned colors red and green). In the following, four or eight colored squares were presented one after another. To achieve the superordinate goal (50 points at the end of each block), subjects had to accept the two target colors shown at the beginning and to reject non-target colors by button press. Two different types of blocks had to be performed. In the first type of blocks, the 'Desire Context' (DC), subjects were allowed to accept the previously conditioned reward stimuli in addition to the two target colors and win bonus points (+10 points). In the second type of blocks, the 'Reason Context' (RC), the conditioned reward stimuli had to be rejected in order to successfully pursue the long-term goal (50 points at the end of the block). So, during the RC, subjects were forced to overcome the tendency to acquire immediate reward (reject the previously conditioned reward stimuli) in order to reach the superordinate long-term goal. This situation therefore constituted a 'desire-reason dilemma' (Diekhof et al, 2012; Diekhof and Gruber, 2010; Trost et al, 2014). For more information, see also Supplementary Figure S1.

\section{Genotyping and Sample Structure}

DNA of all participants was isolated from saliva. Saliva was collected into Oragene saliva DNA kits (DNA Genotek, Kanata, Ontario, Canada) using the Gentra Puregene Blood Kit (Qiagen) with standardized protocols. Genome-wide SNP genotyping was performed using Illumina OmniExpress Genotyping BeadChips according to the manufacturer's standard protocols and using $400 \mathrm{ng}$ of DNA.

EIGENSOFT (Price et al, 2006) was used to identify population outliers based on a principal components analysis. Taken together, these analyses showed that a majority of the subjects included in this study cluster together with HapMap3 European-descent populations. This fact makes unlikely that the results of the study are due to population stratification.

\section{Genotype Group Classification}

Participants were divided into two groups: homozygous major allele carriers $(C / C ; n=131)$ were compared with heterozygous minor allele carriers $(\mathrm{C} / \mathrm{T} ; n=85)$ and homozygous minor allele carriers (T/T; $n=8)$. Gender, age, handedness as well as nicotine, caffeine, and alcohol use (before fMRI) did not differ significantly between groups, see Table 1.

The frequency of the major allele $(C)$ of the MAD1L1 SNP rs 11764590 was 0.77 in the present sample. The observed genotype distribution did not deviate from Hardy-Weinberg equilibrium $\left(X^{2}=1.68, p=0.19,1\right.$ degree of freedom $)$ (Rodriguez et al, 2009).

\section{fMRI Data Acquisition}

fMRI was performed on a 3-Tesla Magnetom TIM Trio Siemens scanner (Siemens Healthcare, Erlangen, Germany) equipped with a standard eight-channel phased-array head coil. First, a T1-weighted anatomical data set with $1 \mathrm{~mm}$ isotropic resolution was acquired. Parallel to the anterior commissure-posterior commissure line, thirty-one axial slices were acquired in ascending direction for fMRI (slice thickness $=3 \mathrm{~mm}$; interslice gap $=0.6 \mathrm{~mm}$ ) using a gradientecho echo-planar imaging sequence (echo time $33 \mathrm{~ms}$, flip angle $70^{\circ}$; field-of-view $192 \mathrm{~mm}$, interscan repetition time $1900 \mathrm{~ms})$.

In 2 functional runs, 185 volumes each were acquired. Subjects responded via button presses on a fiber optic computer response device (Current Designs, Philadelphia, Pennsylvania, USA), and stimuli were viewed through goggles (Resonance Technology, Northridge, California, USA). Presentation Software (Neurobehavioral Systems, Albany, California, USA) was used to present the stimuli in the scanner.

Functional images were preprocessed and analyzed with SPM5 (Statistical Parametric Mapping; www.fil.ion.ucl.ac.uk/ spm/software/spm5/) using a general linear model. The study design was event-related and only correctly answered trials were included in the analysis. 
Linear $t$-contrasts were defined to assess brain activation effects in the two contexts. We analyzed activation effects elicited by the conditioned reward stimuli in the DC and activation effects elicited by the same conditioned stimuli when being presented in the RC. We contrasted the events of conditioned reward stimuli in the DC with those in the $\mathrm{RC}$ to assess the extent of downregulation (suppression) of rewardrelated activation during a competition between the superordinate goal and the proximal reward option in the RC.

Two sample $t$-tests $(\mathrm{C} / \mathrm{C}>\mathrm{T}$ carriers) were performed using single subject contrast images to assess group effects. Age and gender were entered as covariates of no interest. FWE (family-wise error) correction was performed with respect to our a priori regions of interest (small volume analyses of the dopaminergic reward system) and on the whole brain level. Small volume analyses were applied to the bilateral ventral striatum $( \pm 1212-3)$ and the VTA $( \pm 9-21-12)$ (regions of interest, $10 \mathrm{~mm}$ spheres centering a priori coordinates from previous studies) (Diekhof et al, 2012; Diekhof and Gruber, 2010). Further whole-brain genotype group effects were searched for using $p<0.001$, uncorrected.

\section{Psychophysiological Interaction Analysis}

Psychophysiological interaction (PPI) (Diekhof and Gruber, 2010; Friston et al, 1997; Trost et al, 2014) was used to assess functional interactions of the ventral striatum with prefrontal brain regions, especially the anteroventral prefrontal cortex (avPFC), in the 'DRD' situation. Previous studies using the DRD paradigm in healthy subjects had shown a significant negative functional interaction between the ventral striatum and the avPFC in the dilemma situation (Diekhof and Gruber, 2010), while this negative functional interaction had been significantly impaired in bipolar patients in our latter study comparing bipolar patients with healthy controls (Trost et al, 2014). For the PPI analysis, the bilateral ventral striatum (local maximum one-sample $t$-test of all participants in the dilemma situation L ventral striatum -9 123 and $\mathrm{R}$ ventral striatum 1212 6) was selected as seed area $(5 \mathrm{~mm}$ sphere). Small volume analyses using a priori coordinates of the avPFC from our previous studies ( \pm 3051 3, 10 mm sphere; FWE-corrected for small volume; Diekhof and Gruber, 2010; Trost et al, 2014) were carried out to investigate the negative functional connectivity between the bilateral ventral striatum and the avPFC in the 'DRD'.

\section{Beta Value Extraction}

Mean beta estimates were extracted from the bilateral ventral striatum and the VTA using MARSBAR (Brett et al, 2002). Beta extraction for each participant was performed using the coordinates of the local maxima of the whole sample (one sample $t$-test, DC, $p<0.05$, FWE-corrected).

\section{Statistical Analyses}

Behavioral data, personality traits (TCI and BIS) and beta estimates were analyzed using SPSS for Windows (version 21.0, SPSS, IBM, Armonk, New York, USA). Behavioral data: percentage of correctly accepted (DC)/rejected (RC) boni and reaction times were compared between groups. Normal distribution of performance data, TCI, BIS, and beta estimates was tested using the Kolmogorov-Smirnov test and Q-Q plots. Mann-Whitney $U$-tests (in case of nonnormal distribution of scores) and independent sample $t$-tests were used to test for differences between genotype groups (two-tailed significance). The calculation of correlation coefficients was done according to Pearson (Pearson $r$, $p<0.05$, two-tailed significance).

Chi-Square was used to check Hardy-Weinberg equilibrium ( $p>0.05,1$ degree of freedom) (Rodriguez et al, 2009).

\section{RESULTS}

\section{Behavioral Data}

Homozygous major allele carriers and risk allele carriers did not differ significantly with respect to behavioral performance data (Supplementary Table S1). Reaction times were normally distributed and did not differ significantly between groups. Because of significant deviation from normality $(p<0.001)$, group comparisons of percentage of correctly answered bonus trials were done using the nonparametric Mann-Whitney $U$-test. There was no genotype effect on percentage of correctly answered bonus trials (DC and $\mathrm{RC})$.

\section{TCI and BIS Results}

TCI and BIS scores were available for 220 participants $(\mathrm{C} / \mathrm{C}$ $n=128 ; \mathrm{C} / \mathrm{T} \quad n=84 ; \mathrm{T} / \mathrm{T} \quad n=8)$. Differences between genotype groups in TCI and BIS scores and subscales did not reach statistical significance (see Table 1). However, for the TCI subscale harm avoidance there was a trend toward statistical significance between group means of risk allele carriers and non-carriers $(p=0.065)$. Risk carriers showed higher TCI harm avoidance subscale scores than non-carriers

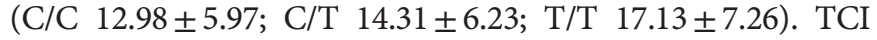
harm avoidance subscale scores were significantly correlated with the number of risk alleles (positive correlation, $r=0.146$, $n=220, p=0.030$, two-tailed significance).

\section{fMRI Data}

In line with our hypothesis, the risk allele carriers exhibited significantly reduced reward-related bottom-up activation in response to the conditioned stimuli in the bilateral VTA, the ventral (and the dorsal) striatum in the DC (Table 2, Figure 1). Small volume analyses using a priori coordinates from our previous studies (Diekhof et al, 2012; Diekhof and Gruber, 2010) confirmed significantly reduced activations in the bilateral ventral striatum and the VTA in the risk allele carriers (FWE-corrected for small volume).

In addition to these activations in mesolimbic dopaminergic brain regions, participants also showed activations in a bilateral fronto-parietal cortical network (associated with salience processing) replicating findings of previous studies (Diekhof et al, 2012; Diekhof and Gruber, 2010; Trost et al, 2014) (Supplementary Table S2).

Within this bilateral fronto-parietal network, activations in the orbitofrontal/insular cortex, the inferior parietal lobule (both regions FWE-corrected across the whole brain), the pre-SMA, and further frontal and parietal cortices were also 
significantly diminished in the risk allele carrier group as compared with homozygous major allele carriers (Supplementary Table S2).

In the RC, both groups showed an (attenuated) BOLD signal in the bilateral VTA and the ventral striatum. In the left VTA and the bilateral ventral striatum, the BOLD signal was significantly diminished (FWE-corrected for small volume) in the risk allele carriers compared with the homozygous major allele carriers (Table 3).

In both groups, activations in the reward task-related fronto-parietal network were found (Diekhof et al, 2012; Diekhof and Gruber, 2010; Trost et al, 2014). The risk allele carriers showed significantly reduced brain activation in the bilateral frontoopercular cortex/insular cortex and the right intraparietal cortex in comparison with the homozygous major allele carriers (Supplementary Table S3).

By contrasting brain activations in the DC with activations in the RC, we assessed the extent of top-down suppression of reward-related cortical and subcortical network activity due to the dilemma. The extent of suppression in the reward regions did not differ significantly between groups. There was a significantly reduced cortical network suppression in the left frontoopercular/anterior insular cortex and the left middle frontal gyrus in the risk allele carriers (Supplementary Table S4).

Table 2 Brain Activations A Priori Regions of Interest 'Desire Context' (DC): C/C $>$ T Carriers

\begin{tabular}{lc}
\hline $\begin{array}{l}\text { A priori regions of } \\
\text { interest }\end{array}$ & $\begin{array}{c}\text { C/C }>\text { T carriers } \\
\text { MNI coordinates (t-values) }\end{array}$ \\
\hline LVTA & $-3-27-24(4.27)$ \\
$R$ VTA & $12-27-15(3.56)$ \\
$L$ ventral striatum & $-2115-3(3.74)$ \\
$R$ ventral striatum & $9156(3.55)$ \\
\hline
\end{tabular}

Abbreviations: L, left; R, right; VTA, ventral tegmental area.

Two sample $t$-test $C / C>T$ carriers, $p<0.05$, FWE-corrected for small volume ( $10 \mathrm{~mm}$ spheres around a priori coordinates bilateral striatum $\pm 1212-3$ and bilateral VTA $\pm 9-21-12$ ).
Additional second-level analyses with TCI harm avoidance subscores as covariates of no interest showed no changes in the main imaging findings (a priori regions of interest) in neither the DC nor the RC (Supplementary Table S5).

\section{PPI Results}

As shown in previous studies (Diekhof and Gruber, 2010), both genotype groups exhibited functional interactions of the ventral striatum with the avPFC in the dilemma situation. The homozygous major allele carriers showed a significant negative functional interaction of the left ventral striatum (seed) with the left avPFC (-24 54 9; $t=3.62 ; p<0.05$, FWEcorrected for small volume, $10 \mathrm{~mm}$ sphere around a priori coordinates). The minor allele carriers did not show this negative functional connectivity, but showed subthreshold positive connectivity between the ventral striatum (seed) and the left avPFC ( $-24510 ; t=2.43 ; p<0.05$, uncorrected). In the group comparison, minor allele carriers showed a significantly reduced negative functional connectivity of the left ventral striatum (seed L ventral striatum: $-9123,5 \mathrm{~mm}$ sphere) with the left avPFC (-27 $513 ; t=3.57 ; p<0.05$, FWE-corrected for small volume, $10 \mathrm{~mm}$ sphere around a priori coordinates).

Table 3 Brain Activations A Priori Regions of Interest 'Reason Context' (RC): C/C > T Carriers

\begin{tabular}{lc}
\hline $\begin{array}{l}\text { A priori regions of } \\
\text { interest }\end{array}$ & $\begin{array}{c}\mathbf{C} / \mathbf{C}>\mathbf{T} \text { carriers } \\
\text { MNI coordinates (t-values) }\end{array}$ \\
\hline$L \vee T A$ & $0-30-2 \mid(3.47)$ \\
$R \vee T A$ & $n . s$. \\
$L$ ventral striatum & $-15 \mid 80(3.35)$ \\
$R$ ventral striatum & $6153(3.99)$ \\
\hline
\end{tabular}

Abbreviations: L, left; R, right; n.S., not significant; VTA, ventral tegmental area. Two sample t-test $C / C>T$ carriers, $p<0.05$, FWE-corrected for small volume ( $10 \mathrm{~mm}$ spheres around $a$ priori coordinates bilateral striatum $\pm 1212-3$ and bilateral VTA $\pm 9-21-12)$.
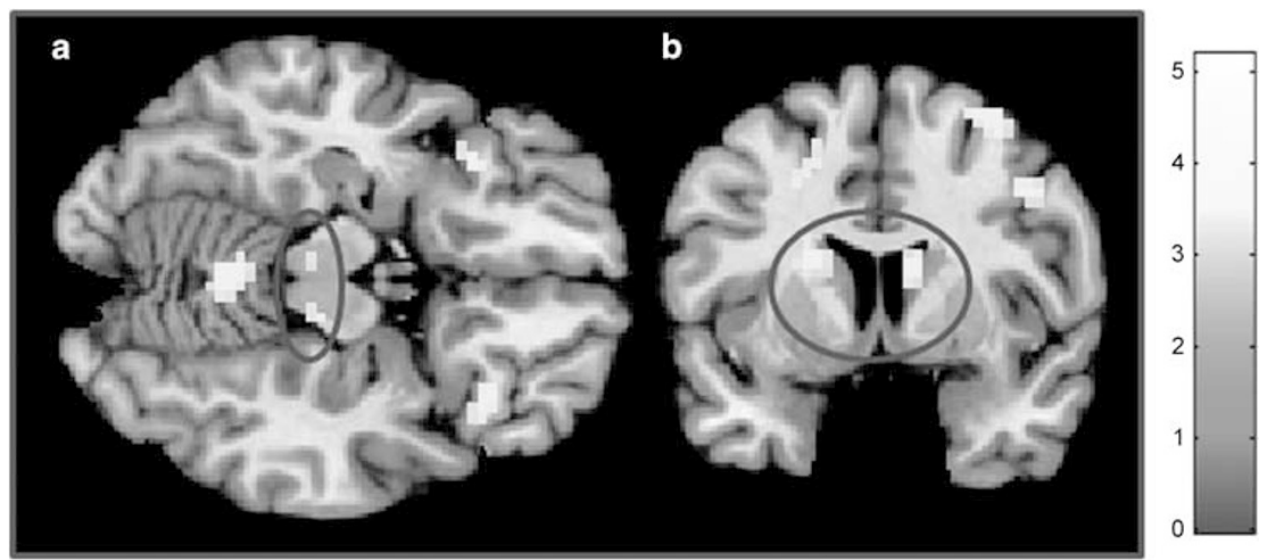

Figure I C/C>T carriers in the DC. (a) Bilateral VTA (blue circle) $(z=-15)$. (b) Bilateral dorsal striatum (blue circle) $(y=6)$. For presentation purposes at $p<0.00 \mathrm{I}$, uncorrected. A full color version of this figure is available at the Neuropsychopharmacology journal online. 


\section{Beta Value Extraction and Exploratory Correlational Analyses}

Mean beta estimates extracted from the bilateral ventral striatum and the VTA in the DC differed significantly between groups. Risk allele carriers showed significantly lower mean beta estimates than homozygous major allele carriers (Supplementary Table S6).

There was no significant correlation of the beta estimates extracted from the bilateral ventral striatum with nicotine or caffeine use.

Post hoc analyses revealed no significant correlation of the TCI harm avoidance subscores with beta estimates extracted from the bilateral ventral striatum or the VTA.

\section{DISCUSSION}

Consistent with our primary hypothesis, young healthy MAD1L1 rs11764590 risk allele carriers showed a reduced bottom-up responsiveness and an altered top-down regulation of the mesolimbic reward system similar to a recently investigated sample of euthymic to mildly depressed bipolar patients, both study samples performing the DRD paradigm (Trost et al, 2014).

The MAD1L1 risk allele carriers showed significantly reduced brain activation in response to conditioned reward stimuli in the VTA and the ventral striatum. This effect was seen bilaterally in the DC when participants were allowed to accept the conditioned reward stimuli and win additional bonus points, but was also present in the RC in the left VTA and the bilateral ventral striatum when subjects had to refrain from accepting the conditioned reward stimuli. In the dilemma situation, a reduced functional connectivity between the avPFC and the ventral striatum in terms of an altered top-down regulation was found in the MAD1L1 risk carriers.

Moreover, risk allele carriers also showed reduced activations in the reward task-related bilateral fronto-parietal network similar to the findings in bipolar patients (Trost et al, 2014). Especially in the anterior insula/frontoopercular cortex, the risk allele carriers exhibited diminished brain activation in response to the conditioned reward stimuli compared with homozygous major allele carriers. This reduced activation occurred in both contexts (in the RC at $p<0.001$, uncorrected). Therefore, we interpret these reduced activations in mesolimbic and cortical regions as a reduced bottom-up response to highly salient, conditioned reward stimuli. Risk allele carriers showed a neurofunctional deficit of their reward responsiveness, in mesolimbic reward areas, but also in task-related cortical regions associated with salience processing.

In schizophrenia research, support for the aberrant salience processing hypothesis is growing (Whitton et al, 2015). Mediated by a dysregulated dopamine transmission in schizophrenia, salience attribution mechanisms are impaired resulting in either inappropriate responses to irrelevant cues or failure to respond adequately to significant events (Kapur, 2003; Whitton et al, 2015).

The finding of an attenuated brain activation in response to reward cues in mesolimbic dopaminergic regions has been multiply replicated in schizophrenia, but is also found in affective disorders (Bogdan et al, 2013; Deserno et al, 2013;
Gradin et al, 2011; Hall et al, 2014; Trost et al, 2014). Drugnaive, first-episode, but also chronic schizophrenia patients show reduced ventral striatal activations during reward anticipation (Deserno et al, 2013); and attenuated positive prediction error signal in the midbrain is another replicated finding in schizophrenia (Deserno et al, 2013; Waltz et al, 2009). Recently, attenuation of striatal activation during reward anticipation was shown in a sample of unaffected first-degree relatives of schizophrenic patients and interpreted as a potential intermediate phenotype for schizophrenia (Grimm et al, 2014).

In bipolar disorder, reward processing is also altered in mesolimbic dopaminergic regions. Recent fMRI studies showed alterations in reward-related striatal and cortical brain activations, state-dependent, but also during euthymia (Abler et al, 2008; Caseras et al, 2013; Nusslock et al, 2012; Trost et al, 2014; Whitton et al, 2015). A number of studies have reported elevated striatal activations in response to rewarding stimuli in hypomanic individuals (O'Sullivan et al, 2011), euthymic (Mason et al, 2014; Nusslock et al, 2012) and manic (Abler et al, 2008) bipolar patients. However, other studies in bipolar patients have shown a reduced reward responsiveness challenging the hypothesis of a general reward hypersensitivity model of bipolar disorder. Decreased striatal activations in response to reward stimuli were found in euthymic to mildly depressed (Trost et al, 2014), depressed bipolar patients (Redlich et al, 2015; Satterthwaite et al, 2015) and euthymic bipolar II/bipolar not otherwise specified patients (Yip et al, 2015); with the latter study reporting differential hypoactivations of both the dorsal and the ventral striatum during reward processing in bipolar disorder (Yip et al, 2015). These results are in line with the present findings of a reduced bottom-up responsivity of striatal regions (dorsal and ventral) in healthy MAD1L1 risk allele carriers.

Furthermore, in our previous study investigating bipolar patients, reduced suppression of brain activation in the dilemma situation was seen in frontal cortical regions (ie, middle frontal gyrus) (Trost et al, 2014), which is also found in the middle frontal gyrus and frontoopercular/ anterior insular cortex in risk allele carriers in the present study (Supplementary Table S2, $p<0.001$, uncorrected). In addition to the finding of reduced functional connectivity in the dilemma situation, this further suggests that not only bottom-up, but also cortically driven top-down mechanisms involved in reward processing are altered in MAD1L1 risk allele carriers.

Risk allele carriers and non-carriers in our study did not differ significantly with respect to demographic or behavioral data. Enhanced trait impulsivity, a characteristic of bipolar disorder (Strakowski et al, 2010), was not found in the risk allele carriers. However, with regard to personality traits associated with MAD1L1, we found a significant correlation of the risk allele with the TCI subscale scores harm avoidance. Subjects scoring high in harm avoidance are described as rather worrying, fearful, shy, and fatigable (Cloninger et al, 1993). Several studies found elevated harm avoidance scores in schizophrenia patients (Jetha et al, 2013; Ohi et al, 2012) and subjects at ultra-high risk for psychosis or experiencing psychotic-like symptoms (Fresán et al, 2015; Nitzburg et al, 2014). With regard to bipolar disorder, harm avoidance was associated with mood episode recurrence in 
bipolar offspring in a longitudinal study (Kemner et al, 2015) and with the overall burden of depressive episodes during lifetime in a sample of bipolar (I and II) and unipolar patients (Zaninotto et al, 2015). Bipolar II patients and patients with major depressive disorder showed higher harm avoidance scores than controls (Zaninotto et al, 2015). In a general population sample, high harm avoidance scores predicted elevated dysphoria rates (Rosenström et al, 2014).

Referring to our imaging findings in association with MAD1L1, the reduced ventral striatal reward responsiveness on the neurofunctional level in the risk allele carriers was not correlated with harm avoidance subscores, but is complemented by findings of personality traits partly overlapping with negative and depressive symptoms in clinical populations. Reduced ventral striatal activation has been associated with apathy in schizophrenia (Kirschner et al, 2015), with anhedonia in depression (Keedwell et al, 2005) and was predominantly present in bipolar patients suffering from depressive symptoms (Redlich et al, 2015; Trost et al, 2014). Therefore, a reduced mesolimbic reward responsivity associated with MAD1L1 may be paralleled by personality traits linked to subthreshold psychopathologic symptoms.

The results of the present study are limited concerning the comparability with other studies investigating the dopaminergic reward system due to the specific fMRI reward paradigm we used. A number of studies cited above used other reward paradigms rather focusing on the neurofunctional underpinnings of reward anticipation and outcome (Caseras et al, 2013; Knutson et al, 2001; Yip et al, 2015) than the neural responses to conditioned reward stimuli under changing conditions with respect to salience of the stimuli. However, despite these methodological differences growing evidence suggests differentially altered reward processing mechanisms in bipolar disorder (with a mesolimbic hyporesponsiveness rather linked to depressive symptoms) and in schizophrenia (failure to respond adequately to significant events) throughout the literature.

Apart from our a priori regions of interest, the findings of reduced cortical activations in the risk allele carriers are limited by the statistical threshold of $p<0.001$ uncorrected. Our objective was to present genotype-associated alterations within the robustly replicated task-related fronto-parietal network linked to salience processing in addition to the subcortical key regions of the mesolimbic reward system.

Another limitation is the fact that only one SNP was investigated in the present study. Our intention was to investigate the possible effects of this one SNP in MAD1L1 which was strongly associated with bipolar disorder (Cichon et al, 2011) and to compare the results to the findings in our bipolar sample (Trost et al, 2014). Moreover, we aimed to integrate our findings into the growing evidence for a phenomenological and genetic overlap of bipolar disorder and schizophrenia (Tamminga et al, 2013). Further studies will be needed to replicate and complement our findings integrating additional genetic data.

Concluding, we suggest that abnormal reward processing can be regarded as a potential endophenotype for both bipolar disorder and schizophrenia. Here, we show that MAD1L1 as a susceptibility gene for both of these genetically overlapping disorders is associated with a decreased bottomup responsiveness of the mesolimbic reward system and related cortical regions involved in the salience network as well as with reduced top-down control processes. By modulating the functionality of these specific subcortical and cortical networks, the MAD1L1 risk variant may increase individual vulnerability for bipolar, affective, or psychotic disorders and contribute to clinical disease manifestation according to the concept of an intermediate phenotype. Furthermore, in parallel to the neurofunctional findings, specific personality traits in the form of higher harm avoidance subscale scores in the risk allele carriers may represent discrete subthreshold symptoms associated with MAD1L1.

\section{FUNDING AND DISCLOSURE}

OG was a honorary speaker for the following companies: Astra Zeneca, Bristol Myers Squibb, Janssen Cilag, Lilly, Servier, and Otsuka. He has been invited to scientific congresses by Astra Zeneca, Janssen Cilag, and Pfizer and has received a research grant from Servier. He reports that these potential conflicts have no relation to the subject of the present study. ST, EKD, HM, HV, BK, CW, MK, PD, and EBB declare that, except for income received from their primary employer, they have no biomedical financial interests or potential conflicts of interest.

\section{ACKNOWLEDGMENTS}

We thank Dr Sergi Papiol for his helpful support with regard to the genetic sample.

\section{REFERENCES}

Abler B, Greenhouse I, Ongur D, Walter H, Heckers S (2008). Abnormal reward system activation in mania. Neuropsychopharmacology 33: 2217-2227.

Bergen SE, O'Dushlaine CT, Ripke S, Lee PH, Ruderfer DM, Akterin S et al (2012). Genome-wide association study in a Swedish population yields support for greater $\mathrm{CNV}$ and $\mathrm{MHC}$ involvement in schizophrenia compared with bipolar disorder. Mol Psychiatry 17: 880-886.

Bogdan R, Nikolova YS, Pizzagalli DA (2013). Neurogenetics of depression: a focus on reward processing and stress sensitivity. Neurobiol Dis 52: 12-23.

Brett M, Anton J-L, Valabregue R, Poline J-B (2002). Region of interest analysis using the MarsBar toolbox for SPM 99. NeuroImage 16: S497.

Calhoun VD, Sui J, Kiehl K, Turner J, Allen E, Pearlson G (2011). Exploring the psychosis functional connectome: aberrant intrinsic networks in schizophrenia and bipolar disorder. Front Psychiatry 2: 75.

Cardno AG, Owen MJ (2014). Genetic relationships between schizophrenia, bipolar disorder, and schizoaffective disorder. Schizophr Bull 40: 504-515.

Caseras X, Lawrence NS, Murphy K, Wise RG, Phillips ML (2013). Ventral striatum activity in response to reward: differences between bipolar I and II disorders. Am J Psychiatry 170: 533-541. Chai XJ, Whitfield-Gabrieli S, Shinn AK, Gabrieli JDE, Nieto Castañón A, McCarthy JM et al (2011). Abnormal medial prefrontal cortex resting-state connectivity in bipolar disorder and schizophrenia. Neuropsychopharmacology 36: 2009-2017.

Cichon S, Mühleisen TW, Degenhardt FA, Mattheisen M, Miró X, Strohmaier J et al (2011). Genome-wide association study identifies genetic variation in neurocan as a susceptibility factor for bipolar disorder. Am J Hum Genet 88: 372-381. 
Cloninger CR, Bayon C, Svrakic DM (1998). Measurement of temperament and character in mood disorders: a model of fundamental states as personality types. J Affect Disord 51: 21-32.

Cloninger C, Svrakic DM, Przybeck TR (1993). A psychobiological model of temperament and character. Arch Gen Psychiatry 50: 975-990.

Deserno L, Boehme R, Heinz A, Schlagenhauf F (2013). Reinforcement learning and dopamine in schizophrenia: dimensions of symptoms or specific features of a disease group? Front Psychiatry 4: 172.

Diekhof EK, Gruber O (2010). When desire collides with reason: functional interactions between anteroventral prefrontal cortex and nucleus accumbens underlie the human ability to resist impulsive desires. J Neurosci 30: 1488-1493.

Diekhof EK, Keil M, Obst KU, Henseler I, Dechent P, Falkai P et al (2012). A functional neuroimaging study assessing gender differences in the neural mechanisms underlying the ability to resist impulsive desires. Brain Res 1473: 63-77.

Fresán A, León-Ortiz P, Robles-García R, Azcárraga M, Guizar D, Reyes-Madrigal F et al (2015). Personality features in ultra-high risk for psychosis: a comparative study with schizophrenia and control subjects using the Temperament and Character Inventory-Revised (TCI-R). J Psychiatr Res 61: 168-173.

Friston KJ, Buechel C, Fink GR, Morris J, Rolls E, Dolan RJ (1997). Psychophysiological and modulatory interactions in neuroimaging. NeuroImage 6: 218-229.

Gottesman II, Gould TD (2003). The endophenotype concept in psychiatry: etymology and strategic intentions. Am J Psychiatry 160: 636-645.

Gradin VB, Kumar P, Waiter G, Ahearn T, Stickle C, Milders M et al (2011). Expected value and prediction error abnormalities in depression and schizophrenia. Brain J Neurol 134: 1751-1764.

Gradin VB, Waiter G, O'Connor A, Romaniuk L, Stickle C, Matthews K et al (2013). Salience network-midbrain dysconnectivity and blunted reward signals in schizophrenia. Psychiatry Res 211: 104-111.

Grimm O, Heinz A, Walter H, Kirsch P, Erk S, Haddad L et al (2014). Striatal response to reward anticipation: evidence for a systems-level intermediate phenotype for schizophrenia. JAMA Psychiatry 71: 531-539.

Hall GBC, Milne AMB, Macqueen GM (2014). An fMRI study of reward circuitry in patients with minimal or extensive history of major depression. Eur Arch Psychiatry Clin Neurosci 264: 187-198.

Hawrylycz MJ, Lein ES, Guillozet-Bongaarts AL, Shen EH, Ng L, Miller JA et al (2012). An anatomically comprehensive atlas of the adult human brain transcriptome. Nature 489: 391-399.

Hill MJ, Donocik JG, Nuamah RA, Mein CA, Sainz-Fuertes R, Bray NJ (2014). Transcriptional consequences of schizophrenia candidate miR-137 manipulation in human neural progenitor cells. Schizophr Res 153: 225-230.

Jetha MK, Goldberg JO, Schmidt LA (2013). Temperament and its relation to social functioning in schizophrenia. Int $J$ Soc Psychiatry 59: 254-263.

Juckel G, Schlagenhauf F, Koslowski M, Filonov D, Wüstenberg T, Villringer A et al (2006). Dysfunction of ventral striatal reward prediction in schizophrenic patients treated with typical, not atypical, neuroleptics. Psychopharmacology (Berl) 187: 222-228.

Kapur S (2003). Psychosis as a state of aberrant salience: a framework linking biology, phenomenology, and pharmacology in schizophrenia. Am J Psychiatry 160: 13-23.

Keedwell PA, Andrew C, Williams SCR, Brammer MJ, Phillips ML (2005). The neural correlates of anhedonia in major depressive disorder. Biol Psychiatry 58: 843-853.

Kemner SM, Mesman E, Nolen WA, Eijckemans MJC, Hillegers MHJ (2015). The role of life events and psychological factors in the onset of first and recurrent mood episodes in bipolar offspring: results from the Dutch Bipolar Offspring Study. Psychol Med 45: 2571-2581.

Kirschner M, Hager OM, Bischof M, Hartmann MN, Kluge A, Seifritz E et al (2015). Ventral striatal hypoactivation is associated with apathy but not diminished expression in patients with schizophrenia. J Psychiatry Neurosci 40: 140383.

Knutson B, Fong GW, Adams CM, Varner JL, Hommer D (2001). Dissociation of reward anticipation and outcome with eventrelated fMRI. Neuroreport 12: 3683-3687.

Lake RJ, Boetefuer EL, Tsai P-F, Jeong J, Choi I, Won K-J et al (2014). The sequence-specific transcription factor c-Jun targets Cockayne syndrome protein $\mathrm{B}$ to regulate transcription and chromatin structure. PLoS Genet 10: e1004284.

Mason L, O'Sullivan N, Montaldi D, Bentall RP, El-Deredy W (2014). Decision-making and trait impulsivity in bipolar disorder are associated with reduced prefrontal regulation of striatal reward valuation. Brain J Neurol 137: 2346-2355.

Nitzburg GC, Malhotra AK, DeRosse P (2014). The relationship between temperament and character and subclinical psychoticlike experiences in healthy adults. Eur Psychiatry 29: 352-357.

Nusslock R, Almeida JR, Forbes EE, Versace A, Frank E, Labarbara EJ et al (2012). Waiting to win: elevated striatal and orbitofrontal cortical activity during reward anticipation in euthymic bipolar disorder adults. Bipolar Disord 14: 249-260.

Ohi K, Hashimoto R, Yasuda Y, Fukumoto M, Yamamori H, Iwase $\mathrm{M}$ et al (2012). Personality traits and schizophrenia: evidence from a case-control study and meta-analysis. Psychiatry Res 198: 7-11.

O’Sullivan N, Szczepanowski R, El-Deredy W, Mason L, Bentall RP (2011). fMRI evidence of a relationship between hypomania and both increased goal-sensitivity and positive outcomeexpectancy bias. Neuropsychologia 49: 2825-2835.

Patton JH, Stanford MS, Barratt ES (1995). Factor structure of the Barratt impulsiveness scale. J Clin Psychol 51: 768-774.

Price AL, Patterson NJ, Plenge RM, Weinblatt ME, Shadick NA, Reich D (2006). Principal components analysis corrects for stratification in genome-wide association studies. Nat Genet 38: 904-909.

Psychiatric GWAS Consortium Bipolar Disorder Working Group (2011). Large-scale genome-wide association analysis of bipolar disorder identifies a new susceptibility locus near ODZ4. Nat Genet 43: 977-983.

Redlich R, Dohm K, Grotegerd D, Opel N, Zwitserlood P, Heindel W et al (2015). Reward processing in unipolar and bipolar depression: a functional MRI study. Neuropsychopharmacology 40: 2623-2631.

Rodriguez S, Gaunt TR, Day INM (2009). Hardy-Weinberg equilibrium testing of biological ascertainment for Mendelian randomization studies. Am J Epidemiol 169: 505-514.

Rosenström T, Jylhä P, Robert Cloninger C, Hintsanen M, Elovainio M, Mantere O et al (2014). Temperament and character traits predict future burden of depression. J Affect Disord 158: 139-147.

Ruderfer DM, Fanous AH, Ripke S, McQuillin A, Amdur RL, Schizophrenia Working Group of Psychiatric Genomics Consortium et al (2014). Polygenic dissection of diagnosis and clinical dimensions of bipolar disorder and schizophrenia. Mol Psychiatry 19: 1017-1024.

Satterthwaite TD, Kable JW, Vandekar L, Katchmar N, Bassett DS, Baldassano CF et al (2015). Common and dissociable dysfunction of the reward system in bipolar and unipolar depression. Neuropsychopharmacology 40: 2258-2268.

Schizophrenia Psychiatric Genome-Wide Association Study (GWAS) Consortium (2011). Genome-wide association study identifies five new schizophrenia loci. Nat Genet 43: 969-976.

Strakowski SM, Fleck DE, DelBello MP, Adler CM, Shear PK, Kotwal $\mathrm{R}$ et al (2010). Impulsivity across the course of bipolar disorder. Bipolar Disord 12: 285-297. 
Subramaniam K, Hooker CI, Biagianti B, Fisher M, Nagarajan S, Vinogradov S (2015). Neural signal during immediate reward anticipation in schizophrenia: Relationship to real-world motivation and function. NeuroImage Clin 9: 153-163.

Sun Q, Zhang X, Liu T, Liu X, Geng J, He X et al (2013). Increased expression of mitotic arrest deficient-like 1 (MAD1L1) is associated with poor prognosis and insensitive to Taxol treatment in breast cancer. Breast Cancer Res Treat 140: 323-330.

Tamminga CA, Ivleva EI, Keshavan MS, Pearlson GD, Clementz BA, Witte B et al (2013). Clinical phenotypes of psychosis in the Bipolar-Schizophrenia Network on Intermediate Phenotypes (B-SNIP). Am J Psychiatry 170: 1263-1274.

Trost S, Diekhof EK, Zvonik K, Lewandowski M, Usher J, Keil M et al (2014). Disturbed anterior prefrontal control of the mesolimbic reward system and increased impulsivity in bipolar disorder. Neuropsychopharmacology 39: 1914-1923.

Tsukasaki K, Miller CW, Greenspun E, Eshaghian S, Kawabata H, Fujimoto $\mathrm{T}$ et al (2001). Mutations in the mitotic check point gene, MAD1L1, in human cancers. Oncogene 20: 3301-3305.

Vessoni AT, Herai RH, Karpiak JV, Leal AMS, Trujillo CA, Quinet A et al (2016). Cockayne syndrome-derived neurons display reduced synapse density and altered neural network synchrony. Hum Mol Genet 25: 1271-1280.
Waltz JA, Schweitzer JB, Gold JM, Kurup PK, Ross TJ, Salmeron BJ et al (2009). Patients with schizophrenia have a reduced neural response to both unpredictable and predictable primary reinforcers. Neuropsychopharmacology 34: 1567-1577.

Whalley HC, Papmeyer M, Sprooten E, Lawrie SM, Sussmann JE, McIntosh AM (2012). Review of functional magnetic resonance imaging studies comparing bipolar disorder and schizophrenia. Bipolar Disord 14: 411-431.

Whitton AE, Treadway MT, Pizzagalli DA (2015). Reward processing dysfunction in major depression, bipolar disorder and schizophrenia. Curr Opin Psychiatry 28: 7-12.

Yip SW, Worhunsky PD, Rogers RD, Goodwin GM (2015). Hypoactivation of the ventral and dorsal striatum during reward and loss anticipation in antipsychotic and mood stabilizer-naive bipolar disorder. Neuropsychopharmacology 40: 658-666.

Zaninotto L, Souery D, Calati R, Di Nicola M, Montgomery S, Kasper S et al (2015). Temperament and character profiles in bipolar I, bipolar II and major depressive disorder: Impact over illness course, comorbidity pattern and psychopathological features of depression. J Affect Disord 184: 51-59.

Zhang X, Huang S, Zhang Z, Wang W (2012). Chapter 10: Mining genome-wide genetic markers. PLoS Comput Biol 8: e1002828.

Supplementary Information accompanies the paper on the Neuropsychopharmacology website (http://www.nature.com/npp) 